\title{
The Institutional Economics of Biodiversity, Biological Materials, and Bioprospecting
}

\author{
By \\ Margaret Polski, Sr. Research Fellow, Indiana University \\ Email: mpolski@indiana.edu
}

Paper prepared for the Second International Workshop: Governance of Biodiversity as a Global Public Good: bioprospection, intellectual property rights, and traditional knowledge, to be held at Louvain-la-Neuve, $5^{\text {th }}$ and $6^{\text {th }}$ February 2004

\author{
January 19, 2004
}

(C) 2004 by author 


\begin{abstract}
The struggle to assign property rights to biological materials and the outcomes of bioprospecting, and to align economic incentives in such a way that biodiversity is conserved presents a number of under-researched analytical issues. This paper uses work in new institutional economics to explore these issues and provide guidance for policymakers who must design appropriate policies and for scholars who are interested in economic organization and regulation. The main findings from the analysis are that biodiversity and biological materials are common pool goods; research on biological materials can but does not necessarily contribute to creating new knowledge and inventions; harvesting biological materials for research and development is a complex contracting situation that involves high risk intertemporal asset transformation and potentially three economically separable goods that have different investment characteristics and contracting requirements; it appears that biodiversity, NHBMs, and bioprospecting are best governed by relationship contracting principals that are designed to fit specific contracting conditions, however empirical analysis of biocontracting experience would improve the reliability and validity of this prediction.
\end{abstract}




\section{Introduction}

Scientific and technological developments in the field of biology have created renewed interest in regulating global biological resources. A prominent outcome of this interest is the Convention on Biological Diversity (CBD), which took effect December 29, 1993. This treaty aims to conserve biodiversity, encourage sustainable use of the components of biodiversity, and assure fair and equitable sharing of the benefits of biodiversity. It has also generated considerable controversy and presents some very interesting challenges for those who study and design economic policies.

At present, 180 countries have committed to work together to implement the provisions of the Convention. ${ }^{i}$ While this group includes countries with a broad array of political economic systems and ideologies, each of the Parties also participates in the World Trade Organization, which explicitly grants property owners the right to earn gains from trade subject to the rule of law.

A number of provisions pose particular challenges for the Parties including those that require national action to protect indigenous knowledge, provide access to biomaterials, assure that the benefits that accrue from the use of biomaterials are shared in fair and equitable ways, facilitate technology transfer, cooperate to advance biotechnological developments, and adopt economically and socially sound measures that act as incentives for conservation and sustainable use. In May 2000, the Counsel of the Parties (COP) decided to establish a working group to develop guidelines on these issues. An ad hoc group was duly constituted and met in October, 2001 in Bonn, Germany. Their work product, the Bonn Guidelines, was subsequently adopted under Decision VI/24 at a meeting of the COP in April, 2002. 
The Bonn Guidelines, which are voluntary, provide a list of elements for material transfer agreements. ${ }^{\text {ii }}$ A key element is an action plan for capacity building for access and benefit sharing. Suggested areas for capacity building include strengthening relevant institutions, valuation of genetic resources and market information, and studies of legislative measures. The Guidelines also anticipate the need to harmonize with related Conventions, such as the International Union for the Protection of New Varieties of Plants (UPOV) and the World Trade Organization's (WTO) agreement on Trade-Related Aspects of Intellectual Property Rights (TRIPS). Finally, they urge the World Property Rights Organization to undertake extensive studies of the impact of intellectual property regimes on genetic resources and scientific research, and to make rapid progress in developing model intellectual property clauses.

Many of the activities that the Parties to the CBD will regulate under the Bonn Guidelines pertain to bioprospecting, which is the search for useful biological materials in microorganisms, plants, fungi, animals, and humans. As scholars and policymakers grapple with regulating bioprospecting activities they are reconsidering classical conceptions of property rights and contracting. For example, some proposals involve unbundling property rights and allocating these rights in different ways. However, many of these proposals are based upon under-researched assumptions about the economic and institutional nature of biodiversity, biological materials, innovation, the nature of contracting, and the strengths and limitations of alternative organizational and governance schemes. 
The struggle to assign property rights to biological materials and the outcomes of bioprospecting and to align economic incentives in such a way that biodiversity is conserved presents a number of analytical issues:

(1) What is the nature of biodiversity? Who is involved in either limiting or sustaining it?

(2) What is the nature of biological materials and biospropecting? How are they connected with biodiversity?

(3) What is the value of different types of biological materials and to whom does this value accrue? Bioprospecting? How do these values change over time, if at all?

(4) What do we know empirically about how to sustain biodiversity and what are the implications of these principles with respect to managing biological materials and biopropecting activities?

(5) What are the principles upon which appropriate regulatory schemes might be designed?

This paper uses work in new institutional economics to explore these issues and provide guidance for policymakers who must design appropriate policies, and for scholars who are interested in economic organization and regulation. The analysis proceeds as follows. Section One deals with the nature of biodiversity and focuses the analysis. The second section analyzes the economic nature of biodiversity and biological materials, and develops the implications for bioprospecting. Section Three identifies some of the contracting problems inherent to bioprosecting and situates these dilemmas in the new institutional economics literature. Section Four concludes. 


\subsection{The Nature of Biodiversity}

Before we can understand how to regulate biological materials and bioprospecting, we must first understand them in the context of sustaining biodiversity. More to the point for this workshop, can we properly conceptualize biological materials as single and distinct types of economic goods or services, such as public informational goods, or do they display their own form of variability?

The CBD defines biological diversity to include "all aspects of variability evident within the living world, including diversity within and between individuals, populations, species, communities, and ecosystems. ${ }^{\text {,iii }}$ This is a very broad definition that potentially includes all nonhuman biological materials (NHBMs) as well as all human biological materials (HBMs) in the world as we know it at a given time. For example, the scope of the CBD potentially includes all the organisms on the Earth and those we discover as we explore our Universe. This is a lot of organisms and a very complex regulatory challenge: in addition to ourselves, organisms exist in natural habitats, in laboratories, in special repositories, and in our homes, offices, and cars - they even travel with us and with our animals.

While the language of the Convention provides a broad scope for action, CBD discussion documents suggest that the Parties are at present focusing on NHBMs and their natural habitats: the most recent report of the Secretariat of the Convention asserts that the diversity of nonhuman species is the most useful measure of biodiversity. ${ }^{\text {iv }}$ The report, which analyzes bacteria, protoctists, animals, fungi, and plants in different types of habitats on the Earth's surface, indicates that around 1.75 million species have been described and formally named. ${ }^{v}$ However, there is reason to believe that several million 
more species exist but remain undiscovered and undescribed. ${ }^{\text {vi }}$ While these species exist in many different types of habitats, they are not evenly distributed over the planet. The most species rich environments holding $60-90 \%$ of all species and accounting for nearly one third of global terrestrial annual net primary production are tropical forests, which extend over about $7 \%$ of the Earth's land surface. Most tropical forests are located in developing countries in Africa, Asia, Central America, Mexico, the Caribbean, and South America.

It is quite difficult to accurately calculate changes in biodiversity because scientific knowledge of the world's species is so incomplete. Scientists estimate that over geological time as a whole, biodiversity has increased even though virtually all the species that have ever existed are now extinct. ${ }^{\text {vii }}$ While extinction of every species is a natural and expected event, evidence from the fossil record indicates that the extinction rate over the past 400 years is $100-200$ times higher than in prior periods. ${ }^{\text {viii }}$ The reliability of this estimate is undermined by the inherent bias in the fossil record however, the general trend is supported by other estimations. Most predictions of the contemporary extinction rate are based on combining estimates of species richness in tropical forests with estimates of rate of loss of these forests. Species extinction is then predicted on the basis of the general species-area relationship, increasing or declining as area increases or declines.

Few actual species extinctions have been observed in tropical forests however, monitoring species in these habitats is quite difficult. Rates of deforestation are high in both absolute and proportional terms, implying a relatively high rate of species extinction. ${ }^{\mathrm{ix}}$ Moreover, there appears to be a link between changes in land cover and 
climate change, which places additional stress on ecosystems and species. Even without deforestation and climate change, species in these habitats are already under stress due to changes in land use; overharvesting; pollution; and incursions by invasive species. Bioprospecting activities may contribute to overharvesting in natural habitats and thus threaten biodiversity or mitigate the effects of overharvesting and thus improve and sustain it.

In summary, for the purpose of grappling with the challenges of implementing the $\mathrm{CBD}$, biodiversity is nonhuman species variation, which is an emergent property of reproduction in natural resource habitats, particularly moist tropical forests in developing countries in Africa, Asia, Central America, Mexico, the Caribbean, and South America. While there is limited numerical evidence that biodiversity is diminishing, we do know that human activities in natural resource habitats can adversely affect species variation when they are poorly managed. So let us now look more deeply at the organization and management of economic activities in natural resources as well as the economic nature of NHBMs and bioprospecting.

\subsection{The Economic Value of Biodiversity}

In economic terms, natural resources are common pool goods: one user's consumption subtracts from the supply available for others yet it is very difficult to exclude users. ${ }^{\mathrm{x}}$ If nonhuman biodiversity emerges from reproduction among living organisms in common pool resources (CPRs) and NHBMs are living organisms, than NHBMs are critical components of CPRs and are themselves common pool goods and vulnerable to degradation. Moreover, if bioprospecting is the search for useful biological materials, than bioprospecting for NHBMs is one of a number of economic activities that 
occur in CPRs and can contribute to resource degradation. This means that the economic nature of NHBMs, bioprospecting, and biodiversity more generally, is a function of how CPR habitats are owned and governed.

There are three important implications of this analysis that are supported by extensive scholarship in institutional economics. The first is that there are important economic differences between common pool, public, club, and private goods. The second implication is that the physical nature of the resource itself creates incentives for users to under-invest in sustainable solutions and conservation activities. A final implication is that if policymakers wish to design and implement effective regulation, they must take these differences and perverse incentives into account.

Since tropical forests are the richest sources of NHBMs, we can use forest resources as a concrete starting point for exploring the economic nature of NHBMs and bioprospecting. Forests are important economic environments that serve a number of productive purposes. In addition to supporting biodiversity and providing a ready environment for bioprospecting, forests are a source of other goods and services that support local economies including:

\section{Goods}

- Wood products, including timber

- Energy, including fuelwood and charcoal

- Non-wood products, e.g. wild game, rattans and fibres, honey, edible and collectable plants, medicinals, aromatics, dyes

\section{Services}

- Recreation 
- Religious practice/spiritual enjoyment

- Climate amelioration

- Regulation of local and regional hydrological cycles

- Mediators in the carbon cycle

- Soil stabilization and watershed protection

Competition for forest products and services is heightened by activities that convert forest area to other economic purposes including cropland, roadways, mining, or residential or industrial development.

At first glance, bioprospectors and biodiversity protectors are not markedly different than other forest users. Bioprospectors must compete with those who harvest other non-wood products, those who harvest wood and energy goods, and those who use the services that forests provide. Biodiversity protectors must compete with other service users as well as those who harvest goods. However, a finer analysis reveals some important differences.

In general, there are three types of bioprospectors who harvest NHBMs: Scientists; Entrepreneurs; and Collectors. Each type has distinct but often overlapping objectives. Scientists bioprospect in connection with doing research. They seek to advance knowledge and solve problems. In the process, they may create new products, processes, or applications; create profitable products; and add to collections of NHBMs. Entrepreneurs bioprospect in connection with building profitable businesses. While their primary objective is to develop profitable products, in the process of doing so they advance knowledge, solve problems, and develop new processes and applications. They may also seek to add to a collection of NHBMs or sell specimens. By comparison, 
Collectors have more limited purposes: they seek new specimens to either expand their own private collection or to sell to others.

\section{Table 1: Bioprospectors and Their Purposes}

\begin{tabular}{|l|c|c|c|}
\hline Purpose & Scientist & Entrepreneur & Collector \\
\hline Advance knowledge & Yes & Yes & No \\
\hline Solve problems & Maybe & Yes & No \\
\hline Create new products, processes, applications & Maybe & Yes & No \\
\hline Develop profitable products & Maybe & Yes & No \\
\hline Expand collection & Maybe & Maybe & Yes \\
\hline Sell specimen to others & No & Maybe & Yes \\
\hline
\end{tabular}

Collectors, like other forest users who harvest goods or consume personal services like recreation and spiritual enjoyment, add little economic value to the material they consume. They invest in harvesting the NHBM for immediate consumption: their own or the person to whom they sell the specimen. The NHBMs they collect are commodities and we can assume that they will continue to harvest until the marginal cost of harvesting exceeds the benefit of doing so. Since the material they harvest has a known, specific use, it is relatively easy to calculate the economic value of this type of bioprospecting and design appropriate incentives. Implementing and sustaining effective policies is another matter entirely and one we must put aside for the time being.

By contrast, Scientists and Entrepreneurs seek to discover the value of the NHBMs they harvest. In the process, they add value to the NHBM, transforming it from one type of asset to another. Their investment includes not only the cost of harvesting, but also the cost of transforming a commodity with a known, specific use into a new 
product with an unknown use. In the process of making this transformation, they create new knowledge that has an economic value that depends upon their investment in studying the NHBM but that is distinct from the economic value of the NHBM and the product they create by applying their new knowledge. In other words, at the successful conclusion of a scientific or entrepreneurial transformation process, there are at least three, potentially separable economic goods:

- The original NHBM (Commodity)

- New Knowledge (Idea)

- Product, Process, or Application (Invention)

I have argued that NHBMs exist in nature as common pool goods. By contrast, new knowledge - the creation of a person's mind - is by nature a private good until or unless the creator shares this knowledge with others. ${ }^{x i}$ That is, consumption has high excludability and high subtractability (the creator can refuse to share new knowledge with others and once new knowledge is consumed by someone other than the creator it is no longer new). Similarly, the products produced from applying new knowledge are by nature private goods until or unless the inventor relinquishes control over production and provision.

NHBMs and the private goods that Scientists and Entrepreneurs create by doing research on NHBMs have different investment characteristics. ${ }^{\text {xii }}$ While NHBMs may be a source of information about life (but are not always), this information does not exist until someone creates it. NHBMs are potential inputs into creative processes that supply new ideas and inventions: they have one value on a stand alone basis, which may be less than or equal to $\mathbf{x}$, and another, unknown value when they are placed in the hands of a 
knowledgeable Scientist or Entrepreneur. Similarly, the new knowledge or an inventive idea that emerges from a creative process is an intangible asset that may be quasi-specific or specific. That is, an idea often has a number of potential applications (quasi-specific) however an invention has just one potential application (specific). The value of new knowledge, which may be equal to or greater than 0 , is not known until it is disseminated and is quite difficult to estimate until further investments are made to apply the idea to address economic demand. However, a new product, process, or application is a tangible, specific asset with one identified use. Its value can be estimated but will not be known in toto until it is fully disseminated. .iii $^{\text {in }}$

In economic terms, knowledge creation is a high risk intertemporal asset transformation process. As Scientists and Entrepreneurs make specific investments to develop knowledge about an NHBM and the NHBM is transformed from one type of asset to another, the focus of attention shifts from the material input to knowledge creation. The ability to appropriate economic value from knowledge creation changes as knowledge develops and is transformed into specific inventions. We know from experience in many scientific fields that this asset transformation process is lengthy, expensive, and risky. It is filled with false starts, dead-ends, and death valleys. Even with the protection of well-enforced intellectual property rights, the appropriability of knowledge assets cannot be protected indefinitely: at some point it diminishes to zero either because intellectual property rights have expired or, more likely, further innovation has made them redundant. ${ }^{\text {xiv }}$

The drug discovery process, which can involve both nonhuman and human biological materials, is a good example of high risk intertemporal asset transformation. It 
takes Scientists and Entrepreneurs on average 10 years to bring a new drug to market at a cost of $\$ 800$ million. Only one of every 5,000 compounds that are investigated in research and development is marketed as a drug, and fewer than $15 \%$ of all drugs that do go to market generate enough revenues to cover the cost of research and development. ${ }^{\mathrm{xv}}$

Stylized, the transformation process works as follows. A Scientist, who possesses general knowledge (a public good with low subtractability and low excludability) and specific knowledge (a private good), has an idea (a private good) about how an immunological process may work. In order to develop this idea, s/he wishes to investigate the properties of a particular organism that thrives in tropical forests. After considerable effort and some direct expense, s/he obtains the required NHBM (a common pool good) and designs and launches a research project.

After several fits and starts, the Scientist is able to demonstrate theoretically that the idea under research shows promise for developing a better understanding of the immunological process. However, additional investment is required to develop this understanding: a team of Scientists with specific knowledge in several disciplines; more lab space; equipment and instrumentation; and so on. In order to obtain this investment, the Scientist must selectively share the specific knowledge s/he has developed and organize an expanded research effort.

After expending further effort, the Scientist obtains additional finance and expands the research project. $\mathrm{S} / \mathrm{He}$ shares unpublished methods and results (private goods) with the new team, at which point they are transformed into jointly owned club goods, which have low subtractability but relatively high excludability. The team makes good progress and begins to publish the results of their research in peer-reviewed 
journals. One of these publications attracts the attention of an Entrepreneur who has an idea (a private good) that the research in which these Scientists are engaged can be successfully applied to combat a disease that greatly threatens public health.

The Entrepreneur, who has successfully launched several new drug therapies and has specific knowledge about how to do this (a private good), gets in touch with the Scientists. After a series of exploratory discussions, the Scientists and the Entrepreneur pool their resources, transforming a combination of private and club goods into a new club good. After a conducting some more research, they discover the potential for a new product. They then form a corporation (a legal person), transfer the idea to the corporation, and obtain finance to fund product development. At this point the idea is transformed from a jointly owned club good to a private good owned by a unified entity.

The corporation obtains a patent that grants it the exclusive right to appropriate economic benefits from the idea that it now owns: the patent expires in 20 years. Research and development continues until the corporation can demonstrate that its prototype satisfies regulatory requirements, a nontrivial process that involves sharing knowledge about the potential product with a large number of people including potential competitors. Once regulatory approvals are obtained, the corporation brings the product to market - another nontrivial process that involves sharing knowledge about the potential product with an even larger number of people, including all known and potential competitors. Twenty years later, the corporation's patent expires regardless of whether it has fully appropriated economic value from the protected invention and the private good this corporation has developed is transformed into a public good. If the corporation does not have other new products to replace this product, it is or will soon be out of business. 
This example demonstrates the economic nature of knowledge creation and the potential role of NHBMs in the knowledge creation process in a particular institutional context. In the first phase, a bundle of public, private, and common pool goods is potentially transformed into an idea about how things work, which is a club good. In the second phase, the idea is potentially transformed into an invention, which is a private good. In the final phase, the invention is transformed into a public good.

Figure 1: The Economic Nature of Knowledge Creation

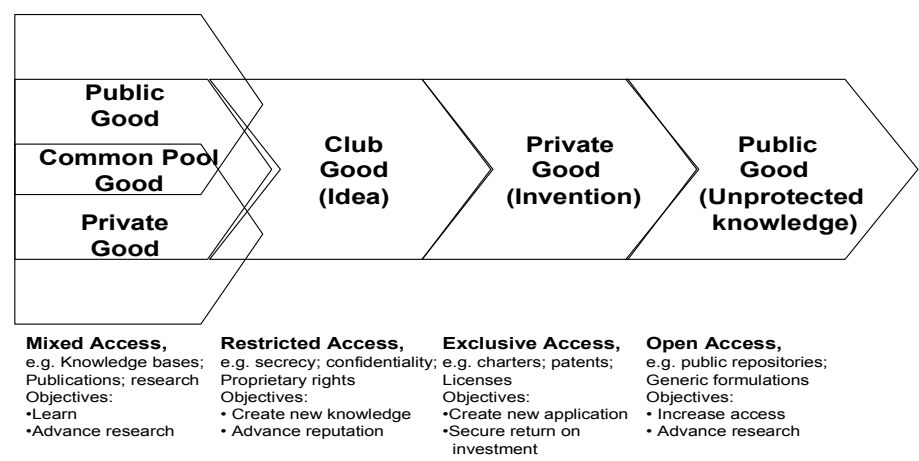

Four implications arise from this analysis: (i) the economic value of NBHMs and knowledge assets change as they are transformed; (ii) transformation requires investment and investment is risky; (iii) as the specificity of assets increase, it is easier to estimate their economic value and risk is reduced; (iv) the institutional context of asset transformation is very important in determining the economic nature of NHBMs and knowledge assets as well as creating incentives to invest in augmenting these values, points that will be explored in more detail in Section Three. 
Figure 2: Economic Value and Asset Specificity

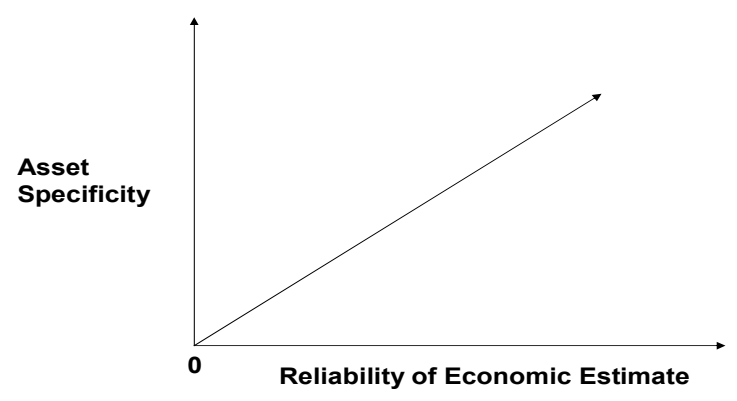

In our stylized example of knowledge creation, the NHBM is a commodity that is studied by a knowledgeable expert in order to create new knowledge. It is a source of inspiration for a creative process: it may or may not be a source of information or a material input into a production process. In fact, a number of outcomes from studying the NHBM are possible.

- Observation reveals no useful information at all

- It provides an interesting analogy: by studying certain properties, the Scientist develops new ideas about how to solve a problem

- Observation reveals useful information that can be obtained from other sources or replicated synthetically

- Observation reveals useful information that cannot be obtained from any other known source or from any other known method

- It has tangible properties that are used in a production process that could be obtained from other sources or replicated synthetically

- It has tangible properties that are used in a production process that cannot be obtained from any other source 
Depending upon what the Scientist or Entrepreneur discovers about the NHBM and how it is used, it may be possible to return it to its habitat unharmed or somewhat disabled, or it may be destroyed. The demand for the NHBM may be quite small or very large. The point is that until we finance in investigations of the use of an NHBM in knowledge creation, we cannot know the relative impact of removing it from its habitat.

Finally, the Scientist or Entrepreneur locates and studies the NHBM to advance theoretical and practical knowledge in immunology. In the process, s/he may identify and describe a living organism. This information may or may not already exist - recall that it is widely believed that millions of species remain undefined and undescribed. While this is not the intended purpose of the research, it is yet another product of the research effort that has potential value. However, its value may be in fields that are outside the Scientist's or Entrepreneur's area of expertise. Moreover, there is a potential conflict among these different uses, e.g. keeping this knowledge secret may give the Scientist or Entrepreneur a competitive advantage that $\mathrm{s} / \mathrm{he}$ is not willing to relinquish at all or until some period of time has passed.

In summary, determining the economic nature and hence the economic value of NHBMs requires a great deal of specific knowledge about NHBMs, their importance in their habitats, their supply, and their potential uses. Given the state of knowledge about biodiversity, it is premature to describe and treat all living nonhuman organisms as a single class of economic goods. It is particularly misleading to conceptualize them as public information goods. Until we have evidence to the contrary, the most sensible starting point is to classify biodiversity and the components of biodiversity as common pool goods that are tangible, specific assets. If we wish to design incentives to achieve 
the objectives of the $\mathrm{CBD}$, we must focus on solving the contracting problems associated with sustainable CPR management, and if we wish to transform CPR assets into ideas and inventions, we must focus on solving the contracting problems that arise in high risk intertemporal asset transformation.

\subsection{Contracting Dilemmas}

Let us begin analyzing contracting dilemmas by reviewing empirical evidence on governing forest resources and see what this suggests with respect to governing CPR asset transformation.

\subsection{Governing Common Pool Resources}

While there is still much to be learned about governing common pool resources, thanks to the extensive empirical work of Lin Ostrom and her colleagues, we can extract general principles for sustainable governance based on institutional experiments conducted in a wide range of resources located all over the world.

In a recent article in Science, Dietz, Ostrom, and Stern argue from evidence on forest resources that there is no best way to own or effectively govern common pool resources. ${ }^{\text {xvi }}$ This is because there is considerable variation among CPRs and the conditions within which they are situated. Moreover, many of our most important resource systems are continually evolving and they are subject to global pressures such as climate change or international trade - tropical forests are a prominent example. These types of resources require complex, nested forms of governance that include contracting within and among self-governing user groups and other interested parties; local, national, and regional governments; and international bodies. 
Dietz, et al., propose that the best way to meet the challenges involved in sustaining contemporary forest resources is to devise flexible and adaptable institutional arrangements, which are congruent with specific resource conditions, and that address the following issues:

- Information. High quality, reliable information about the state of the resource is required to effectively regulate the resource, including information about stocks, flows, processes, interactions, and human activities; uncertainty; and values. Information should be congruent with the scale of environmental events and decisions, as well as decision-makers' needs.

- Consultation and Conflict Management. Inclusive, participatory processes are more likely to produce appropriate information, decisions, commitment, and cooperation than more authoritarian processes.

- Rule Compliance. The most effective approach to insuring rule compliance is to consistently deliver progressive sanctions that include a variety of informal and formal mechanisms, which requires adequate resources for monitoring and enforcement. Mechanisms like Tradable Environmental Allowances (TEAs) can contribute to effective compliance if a number of conditions are met, e.g. stocks and flows are predictable; users/producers are relatively homogeneous and the size of the group is controlled; permits are clearly defined and fully exchangeable, and they are used in combination with community-based systems. Voluntary approaches can be effective when combined with other strategies that provide incentives for compliance. 
- Infrastructure. Appropriate physical, technological, and institutional infrastructure is required to create accountability. It also facilitates conflict resolution and coordination.

- Change. Codified rules, which rely on current knowledge and static conditions, are less effective in coping with long-run change than more general principles that address objectives and consequences and allow for adaptation.

The principles that Dietz, et al. extrapolate from data on forest resource management experience are remarkably consistent with Williamson's theory of efficient transacting: the investment dimensions of most CPR transactions involve considerable specificity and high uncertainty and hence require relational contracting, using either bilateral or unified mechanisms. ${ }^{\text {xvii }}$ While some CPR transactions may lend themselves to market (classical contracting) or trilateral (neoclassical contracting) governance mechanisms such as TEAs and voluntary regulation, Dietz, et al. suggest that for these mechanisms to be effective, the investment characteristics of the CPR transaction must fit these particular governance structures and contracting must be nested in relational contracting structures.

\subsection{Asset Transformation}

Despite the plethora of contracting arrangements suggested by empirical work on CPR governance, there appears to be a dearth of comparable empirical knowledge of bioprospecting and knowledge creation using NHBMs. Until we have data, the best we can do is to apply theory to stylized facts and speculate about appropriate governance principles. 
Returning to the example of asset transformation developed in Section Two, let us decompose this process into discrete transactions and apply Williamson's classification scheme for organizing efficient governance. ${ }^{\text {xviii }}$ Table 2 shows the decomposition and theoretical application. Several observations can be made from this analysis. First, the transactions described in our stylized example are nested in other transactional situations, e.g. transactions between a CPR owner and a Knowledge Creator are potentially influenced by contracting between the CPR owner and the Nation State within which the resource resides; the Nation State and the CBD; and the CBD and other International Bodies such as the WTO or the UPOV. Similarly, transactions between a Corporation and a Patent Provider, Commercial Investors, and a Regulator are potentially influenced by contracting with Polities.

Second, there are many potential transactions that are not addressed by our stylized facts that occur in the naturally occurring world, e.g. we assume our Scientists are solo agents but they may be employed by a university, a government research lab, a private research lab, or some other type of organization that has an interest in their activities; our facts do not address the agreements among the Scientists or the Scientists and the Entrepreneur with respect to ownership and control of their joint research products; our facts do not address the agreements between the Scientists, the Entrepreneur, and the Corporation to which they transfer their Invention and for which they conduct additional research and development.

Third, in toto, this is a very complex governance situation that cannot be addressed with a single governance mechanism. Instead, it would appear to require a strong sensitivity to particular transactions and a mix of different mechanisms. 
Fourth, many of these asset transformation transactions involve high degrees of asset specificity and uncertainty. In Williamson's world, relational contracting principles, which are consistent with the principles Dietz, et al. articulate for good CPR governance, are the best guide for developing appropriate governance mechanisms.

\subsection{Conclusion}

This paper explores the analytical issues involved in regulating bioprospecting and the use of nonhuman biological materials to advance scientific knowledge and develop new products, processes, and applications that address human health needs and have commercial value. The analysis is sufficiently general that is can provide guidance for policymakers in countries that are Parties to the Convention on Biodiversity, those in countries that are not Parties to the Convention, and others who are interested in these matters including institutional scholars.

The main findings from this analysis are:

(1) Biodiversity is an emergent property of common pool resource management, mainly tropical forests located in developing countries in Africa, Asia, Mexico, the Caribbean, and Central and South America

(2) Bioprospecting for nonhuman biological materials, which is the search for useful biological materials in microorganisms, plants, fungi, animals, is one of a number of economic activities that take place in common pool resources, particularly tropical forests.

(3) Nonhuman biological materials (NHBMs), which are tangible, specific assets that are harvested from common pool resources, are common pool goods. 
(4) Research on NHBMs can but does not necessarily contribute to creating new knowledge and inventions.

(5) Harvesting NHBMs for research and development is a complex contracting situation that involves high risk intertemporal asset transformation and potentially three economically separable goods that have different investment characteristics and contracting requirements: the NHBM; an Idea; and an Invention.

(6) It appears that biodiversity, NHBMs, and bioprospecting are best governed by relationship contracting principals that are designed to fit specific contracting conditions, however empirical analysis of biocontracting experience would improve the reliability and validity of this prediction. 
Table 2: BioContracting

\begin{tabular}{|l|l|l|l|l|}
\hline Parties & Asset & Specificity & Frequency & Uncertainty \\
\hline CPR Owner \& Nation State & CPR & High & High & High \\
\hline Nation State \& CBD & CPR & High & Low & High \\
\hline CBD \& Other International Bodies & CPR & High & Low & High \\
\hline CPR Owner \& Knowledge Creator & NHBM & Low & Low & High \\
\hline Knowledge Creator \& Knowledge Investor & Idea & Low & Medium & High \\
\hline Knowledge Creator \& Other Knowledge & Idea & Medium & High & High \\
\hline Creators & & & & \\
\hline Knowledge Creators \& & & Medium & Low & Medium \\
\hline Publisher & Idea & & & \\
\hline Knowledge Creators \& Entrepreneur & Idea & Medium & High & High \\
\hline Knowledge Creators \& Patent Provider & Invention & High & Low & High \\
\hline Knowledge Creators \& Corporation & Ideas, & Low-High & High & High \\
\hline Regulator \& Polity & Invention & & High & \\
\hline Corporation \& Commercial Investors & Ideas, & Low-High & Low & High \\
\hline & Invention & & Medium & Medium- \\
\hline Invention & Invention & High & High \\
\hline
\end{tabular}

\footnotetext{
${ }^{\mathrm{i}}$ The complete Convention is available at http://www.biodiv.org. The Cartagena Protocol on Biosafety, which was adopted in January 2000 , by the member countries to the Convention, regulates the transboundary movement of living modified organisms (LMOs) resulting from biotechnology. It supplements the Convention by providing a harmonized set of international rules and procedures to ensure that countries have the relevant information to enable them to make informed decisions about importing LMOs. Information about the Protocol is available at http://www.biodiv.org/biosafety.

${ }^{\text {ii }}$ Decision VI/24, which includes the Bonn Guidelines, is available at http://www.biodiv.org.
} 
iii Convention on Biological Diversity.

iv See Secretariat of the Convention on Biological Diversity. 2001. "Global Biodiveristy Outlook." United Nations Environment Programme. November. This report is available at http://www.biodiv.org. ${ }^{v}$ Ptotoctists include all the eukaryotic single-celled microrganisms as well as certain multi-celled organisms, such as kelp, that don't belong to the plant, animal or fungi kingdoms. There thousands of species of these organisms, all of them aquatic: they include the amoebas, algae, seaweeds, slime molds, ciliates, diatoms, paramecia, forams and many others.

${ }^{v i}$ The World Resources Institute estimates that more than 10 million species inhabit the Earth.

${ }^{\text {vii }}$ Secretariat of the Convention on Biological Diversity (November, 2001).

viii Ibid.

${ }^{\text {ix }}$ Ibid. By contrast, temperate forests, which are mostly located in developed countries, are losing area at lower rates or showing an increase in area.

${ }^{x}$ For an overview of this taxonomy and its relevance for institutional analysis and design, see Polski, Margaret and Elinor Ostrom. 1999. "An Institutional Framework for Policy Analysis and Design.”

Workshop in Political Theory and Policy Analysis Working Paper W98-27. For a more complete analysis of CPR governance issues, see the extensive work of Elinor Ostrom.

${ }^{\mathrm{xi}}$ For an analysis of scholarly information as a common pool resource, see Hess, Charlotte and Elinor Ostrom. 2003. "Ideas, Artifacts, and Facilities: Information as a Common Pool Resource. Law and Contemporary Problems. School of Law. Duke University. Vol. 66. Nos. 1\&2. Winter/Spring.

${ }^{x i i}$ For an analysis of the role of investment characteristics in institutional analysis and design, see Williamson, Oliver E. 1985. The Economic Institutions of Capitalism. New York, NY: The Free Press. xiii It may be that additional uses can be discovered for a particular product, process, or application, however, discovering these new uses requires new investment in specific knowledge and so we can think of this as an iteration of the asset transformation process.

${ }^{\text {xiv }}$ For an analysis of some of the dilemmas posed by this process, see Polski, Margaret. 2000. "Sustaining Innovation and Growth in Research Intensive Industries." Discussion Paper prepared for Board on Science, Engineering, Technology, and Economic Policy. August.

${ }^{\mathrm{xv}}$ Standard and Poor. 2003. Industry Survey on Healthcare: Pharmaceuticals. June 26.

${ }^{x v i}$ Dietz, Thomas, Elinor Ostrom, and Paul C. Stern. 2003. "The Struggle to Govern the Commons." Science. December 12; 302:1907-1912. While Lin Ostrom has produced an extensive body of work on CPR governance with a number of colleagues, this article is of particular interest for this analysis because the arguments are based upon recent empirical work on global forest resources.

${ }^{x v i i}$ The theory of efficient governance is described in Chapter 3 in Williamson, Oliver E. 1985. The Economic Institutions of Capitalism. New York, NY: The Free Press.

xviii Ibid. 